\title{
The effect of semen collection location and time to processing on sperm parameters and early IVF/ICSI outcomes
}

\author{
Caitlin R. Sacha ${ }^{1}$ (D) Stylianos Vagios ${ }^{1} \cdot$ Karissa Hammer $^{1} \cdot$ Victoria Fitz $^{1} \cdot$ Irene Souter $^{1} \cdot$ Charles L. Bormann $^{1}$ \\ Received: 24 November 2020 / Accepted: 23 February 2021 / Published online: 11 March 2021 \\ (C) The Author(s), under exclusive licence to Springer Science+Business Media, LLC, part of Springer Nature 2021
}

\begin{abstract}
Purpose We aimed to assess whether home collection and increased time to semen processing are associated with altered sperm parameters, fertilization rates (FR), day 5 usable quality blastocyst development rates (D5-UQBR), or pregnancy rates (PR) in patients undergoing IVF/ICSI.

Methods This was a retrospective cohort study of patients undergoing IVF/ICSI before the coronavirus disease 2019 (COVID-19) pandemic ("clinic" collection, $n=119$ ) and after COVID-19 ("home" collection, $n=125$ ) at an academic fertility practice. Home collection occurred within $2 \mathrm{~h}$ of semen processing. Patient sperm parameters, FR (\#2PN/MII), D5-UQBR (\# transferable and freezable quality blastocysts/\# 2PN), and PR in fresh transfer cycles were compared between clinic and home groups with $t$-tests. The association between time to processing on outcomes was assessed with regression modeling, controlling for potential confounders.

Results Mean male age was 37.9 years in the clinic group and 37.2 years in the home group $(p=0.380)$. On average, men were abstinent for 3.0 days (SD 1.7) in the clinic group and 4.1 days (SD 5.4) in the home group $(p=0.028)$. Mean time to semen processing was $35.7 \mathrm{~min}$ (SD 9.4) in the clinic group and $82.6 \mathrm{~min}$ (SD 33.8) in the home group $(p<0.001)$. There was no association between collection location and increased time to processing on sperm motility, total motile count, FR, D5-UQBR, or PR.

Conclusions Our data suggest that increased time to processing up to $2 \mathrm{~h}$ with home semen collection does not negatively impact sperm parameters or early IVF/ICSI outcomes.
\end{abstract}

Keywords COVID-19 $\cdot$ IVF $\cdot$ Semen processing $\cdot$ Timing $\cdot$ Sperm parameters $\cdot$ Embryo development

\section{Introduction}

The standard approach to semen collection and processing for use in in vitro fertilization (IVF) and intracytoplasmic sperm injection (ICSI) adopted by fertility clinics in accordance with World Health Organization (WHO) recommendations involves a private collection room adjacent to the andrology laboratory [1]. This setup minimizes temperature changes and length of time, both thought to be potentially detrimental to sperm quality, between collection and processing [1-4].

CRS and SV should be considered joint first authors.

Caitlin R. Sacha

CSACHA@ @artners.org

1 Massachusetts General Hospital Fertility Center, Division of Reproductive Endocrinology and Infertility, Department of Obstetrics, Gynecology, and Reproductive Biology, Massachusetts General Hospital and Harvard Medical School, 32 Fruit Street-Yawkey 10A, Boston, MA 02114, USA
Home collection within an hour of processing for diagnostic semen analyses is noted in the WHO manual to be an acceptable alternative in "exceptional" cases [1].

However, the limited data regarding the effect of location and time between semen collection and processing for fertility treatments reveal conflicting findings. In their prospective cohort study on the effect of collection location on semen parameters, Shetty Licht et al. found no significant differences between home and clinic collections in the same men undergoing diagnostic semen analysis or IUI [5]. Similarly, a recent randomized trial illustrated no differences in semen analyses between men collecting at home or at clinic [6]. A crosssectional study by Elzanaty and Malm of 379 semen samples observed that home collections had significantly higher sperm concentration, motility, and total motile count compared to clinic collections [7]. A recent work by Stimpfel et al. analyzing home vs. clinic collection in fresh IVF/ICSI cycles similarly found increased sperm concentration and motility in home collections, with an improvement in usable embryo rate as well [8]. Importantly, time between semen collection and 
processing was not analyzed in these studies. In their analysis of 62 couples undergoing IUI, Yavas and Selub found that shorter time intervals between semen collection and IUI were associated with improved pregnancy outcomes in women who received human menopausal gonadotropin ( $\mathrm{hMG}$ ), but not in women who received clomiphene citrate (CC); they recommended performing IUI within 90 min of semen collection in clinic for patients treated with hMG [9]. In contrast, in a larger study of 633 IUI cycles, Song et al. observed no differences in sperm parameters or PR between clinic and home collections with longer time to processing, regardless of the presence of male factor or the transport season [10].

Due to the coronavirus disease 2019 (COVID-19) pandemic, our center established a protocol for home semen collection in order to maintain social distancing and reduce the risk of exposure to our patients. Home collection significantly lengthened the time between collection and processing. We aimed to assess the effect of collection location and time to processing of semen samples on sperm parameters, fertilization rate (FR), day 5 usable quality blastocyst development rate (D5-UQBR), and pregnancy rate (PR), in couples undergoing IVF/ICSI.

\section{Materials and methods}

\section{Study design}

This retrospective cohort study was approved by the Massachusetts General Hospital (MGH)/Partners Institutional Review Board. Patients who underwent autologous IVF cycles with oocyte retrievals between January 1, 2020 , and July 31,2020 , were included. For a subgroup analysis comparing clinic to home collection within the same patient, we included data from their pre-COVID IVF cycle (2016-2020). Our center shut down due to the COVID-19 pandemic and performed no onsite oocyte retrievals between March 24, 2020, and June 5, 2020, per the state of Massachusetts policies for elective procedures. The only protocol change in our IVF laboratory after re-opening consisted of home semen collection.

\section{Sperm collection and processing for IVF/ICSI}

In all cycles, semen samples were produced by masturbation without the aid of lubricants. Men were instructed to abstain from ejaculating for 2 to 7 days prior to the scheduled oocyte thaw or retrieval. Semen collection at our center prior to the COVID-19 shutdown occurred in a collection room adjacent to the andrology laboratory ("clinic" collection), which facilitated processing within approximately $30 \mathrm{~min}$ of sample production. Samples produced onsite were liquefied at $37^{\circ} \mathrm{C}$ for 20-60 min before analysis.
Patients collecting specimens offsite after the COVID-19 shutdown were asked to transport their specimens to our center within $2 \mathrm{~h}$ of collection and to keep their sample at body temperature. The samples were delivered by the patient with a valid form of identification. Home collection samples were processed within $20 \mathrm{~min}$ of receiving the specimen. If they could not be processed immediately, they were held in a $37^{\circ} \mathrm{C}$ incubator. Sample temperature was not assessed prior to processing. Sperm count and motility were assessed with a computer-aided semen analysis system (CASA) (HamiltonThorne Biosciences, Ceros, Version 14 ) at $37^{\circ} \mathrm{C}$.

Semen samples were processed for IVF and ICSI using a density gradient sperm isolation technique. Specimens were layered over a $90 \%$ Isolate gradient (Isolate ${ }^{\circledR}$, Fujifilm) and centrifuged for $20 \mathrm{~min}$ at $300 \mathrm{~g}$. Following centrifugation, sperm pellets were suspended with $10 \mathrm{~mL}$ of multipurpose handling medium-complete (MHM, Fujifilm) and centrifuged for $10 \mathrm{~min}$ at $300 \mathrm{~g}$. This wash step was repeated two times for each specimen. The final sperm pellet was overlaid with human tubal fluid (HTF, Fujifilm), supplemented with 5\% human serum albumin (HSA, Fujifilm), and placed in an incubator set at $37^{\circ} \mathrm{C}$ with an atmosphere of $5 \% \mathrm{O}_{2}$ and $6.5 \% \mathrm{CO}_{2}$ until insemination.

\section{Ovarian stimulation protocols}

The study patients underwent a standard infertility work-up as previously described [11-14]. Protocols for controlled ovarian hyperstimulation included luteal-phase gonadotropin-releasing hormone $(\mathrm{GnRH})$ agonist, $\mathrm{GnRH}$ antagonist downregulation, or GnRH agonist flare protocols, with follicular synchronization and pituitary downregulation with oral contraceptive pills as clinically indicated. During treatment with recombinant gonadotropins, patients were serially monitored with transvaginal ultrasound to assess follicular measurements and endometrial thickness and serum estradiol $\left(\mathrm{E}_{2}\right)[14]$. Once at least three follicles reached $16 \mathrm{~mm}$ or more in diameter and the $\mathrm{E}_{2}$ level was $>600 \mathrm{pg} / \mathrm{mL}$, intramuscular human chorionic gonadotropin (hCG) or subcutaneous leuprolide acetate was administered to induce oocyte maturation. The patients underwent a transvaginal ultrasound-guided oocyte retrieval $36 \mathrm{~h}$ later [15].

\section{Fertilization and embryo transfer protocols}

Couples underwent IVF with conventional insemination or IVF with ICSI as clinically indicated between 3 and $5 \mathrm{~h}$ post oocyte thaw or retrieval. Fertilization was assessed between 16 and $18 \mathrm{~h}$ after insemination. Normally fertilized, 2PN stage zygotes were cultured in Continuous Single Culture NXComplete (CSC-NX) media, overlaid with mineral oil (Ovoil, Vitrolife), and cultured in a benchtop incubator set at $37^{\circ} \mathrm{C}$ with an atmosphere of $5 \% \mathrm{O}_{2}$ and $6.5 \% \mathrm{CO}_{2}$. 
Fresh embryo transfer was performed on day 3 or day 5 based on the number of embryos available and their quality according to clinic protocol. High-quality blastocysts, defined as $\geq$ stage 3 with a grade A or B inner cell mass and trophectoderm (Gardner et al., 2000), were eligible for cryopreservation. Intramuscular progesterone, $50 \mathrm{mg}$ per day, was begun on the day after oocyte retrieval for those undergoing a fresh transfer and continued until 10 weeks of gestation if conception occurred. Positive pregnancy test was defined as a serum $\beta$-hCG level $>6 \mathrm{mIU} / \mathrm{mL}$, typically measured 17 days (range 15-20 days) after oocyte retrieval. Implantation rate (IMPR) was defined as the number of sacs seen on ultrasound per number of embryos transferred. Clinical information was abstracted from the patient's electronic medical record by the research staff.

\section{Outcome measures}

Our primary outcomes of interest included sperm parameters (concentration, motility, total motile count, and forward progression score), FR per cycle (number of 2 pronuclear stage embryos/number metaphase II oocytes), D5-UQBR per cycle (number of transferable and freezable quality blastocysts/ number 2 pronuclear stage embryos), and PR (number positive pregnancy tests/number of embryo transfers) in fresh transfer cycles.

\section{Statistics}

Our outcomes were compared between clinic and home collections with independent and paired samples $t$-tests, as indicated. The association between time from semen collection to processing, sperm parameters, FR, and D5-UQBR was assessed with linear regression modeling. The association between time to processing and PR was assessed with logistic regression modeling. The models were controlled maternal age, paternal age, days of abstinence, fertilization method, and day of transfer, as applicable. The number of embryos transferred was additionally controlled when assessing PR. A $p$-value $<0.05$ was considered statistically significant. Statistical analysis was conducted in SPSS version 21.0 (Armonk, NY).

\section{Results}

\section{Study population}

Study population characteristics are shown in Table 1. Mean female age was 36.6 years (SD 3.9) in the clinic group and 36.8 years (SD 4.3) in the home group $(p=0.745)$. Male age was 37.9 years (SD 5.3) in the clinic group and 37.2 years (SD $6.1)$ in the home group $(p=0.380)$. Male infertility, alone or in combination with other factors, was a common infertility diagnosis, present in $52.3 \%$ of the clinic group and $44.8 \%$ of the home group (Table 1). On average, men were abstinent for 3.0 days (SD 1.7) in the clinic group and 4.1 days (SD 5.4) in the home group $(p=0.028)$. Mean time to semen processing was $35.7 \mathrm{~min}$ (SD 9.4) in the clinic group and $82.6 \mathrm{~min}$ (SD 33.8) in the home group $(p<0.001)$.

Semen analysis parameters were similar between the clinic and home groups, as shown in Table 1 . There were no differences in FR (clinic $77.6 \%$ vs. home $77.9 \%, p=0.939$, Table 1). When evaluated separately, there were no differences in FR between the clinic and home groups amongst those who underwent conventional IVF or those who underwent ICSI (Table 1). D5-UQBR was $51.6 \%$ in the clinic group and $55.5 \%$ in the home group $(p=0.225$, Table 1$)$. There was a higher percentage of day 3 transfers in the clinic group (42.2\%) compared to the home group $(24.7 \%, p=$ 0.031 , Table 1). Number of embryos transferred, implantation rate, and pregnancy rate were similar between the two groups (Table 1).

\section{Effect of collection location on sperm parameters and embryology outcomes}

Compared to cycles with clinic collection, there was no effect of home collection on sperm parameters, FR, D5-UQBR, or PR, as shown in Table 2.

\section{Effect of time to semen processing on sperm parameters and embryology outcomes}

Amongst all cycles, there was no effect of increased time to semen processing on sperm motility (coefficient 0.044 $(-0.039,0.126), p=0.302)$, total motile count (coefficient $0.309(-0.09,0.707), p=0.128)$, FR (coefficient $0.000(0$, $0.001), p=0.306)$, D5-UQBR (0 ( $-0.001,0.001), p=$ $0.698)$, or PR (1.008 (0.998, 1.018), $p=0.137$, Table 3). Similarly, time to processing had no effect on these outcomes in the clinic and home groups individually or in the adjusted models (Table 3). Although there was an increase of 0.002 forward progression score in all cycles with increased time to processing $(p=0.042)$, the score was not affected by time to processing when analyzed separately in the clinic and home groups (Table 3). These findings remained consistent in the adjusted models (Table 3 ).

\section{Effect of collection location and time to semen processing on sperm parameters and embryology outcomes within the same patient}

We performed an additional subgroup analysis comparing outcomes from cycles with clinic collection to those with home collection within the same patient. There were 42 
Table 1 Demographic and clinical characteristics of total cohort*
Table 2 Association between collection at home and sperm parameters and embryology outcomes

\begin{tabular}{|c|c|c|c|}
\hline & Clinic $(N=119)$ & Home $(N=125)$ & $p$-value \\
\hline Age female, yrs & $36.6(3.9)$ & $36.8(4.3)$ & 0.745 \\
\hline Age male, yrs & $37.9(5.3)$ & $37.2(6.1)$ & 0.380 \\
\hline \multicolumn{4}{|l|}{ Infertility diagnosis } \\
\hline Male & $26(21.2 \%)$ & $22(17.6 \%)$ & \multirow[t]{9}{*}{0.290} \\
\hline Endometriosis & $3(2.5 \%)$ & $2(1.6 \%)$ & \\
\hline Diminished ovarian reserve & $8(6.7 \%)$ & $18(14.4 \%)$ & \\
\hline Tubal & $5(4.2 \%)$ & $4(3.2 \%)$ & \\
\hline Uterine & $3(2.5 \%)$ & $0(0 \%)$ & \\
\hline Ovulatory disorders & $5(4.2 \%)$ & $4(3.2 \%)$ & \\
\hline Other & $11(9.2 \%)$ & $19(15.2 \%)$ & \\
\hline Unexplained & $22(18.5 \%)$ & $22(17.6 \%)$ & \\
\hline Combined & $36(30.3 \%)$ & $34(27.2 \%)$ & \\
\hline Days of abstinence & $3.0(1.7)$ & $4.1(5.4)$ & 0.028 \\
\hline Time to processing, $\min$ & $35.7(9.4)$ & $82.6(33.8)$ & $<0.001$ \\
\hline \multicolumn{4}{|l|}{ Fertilization method } \\
\hline IVF & $8(6.7 \%)$ & $11(8.8 \%)$ & \multirow[t]{2}{*}{0.545} \\
\hline ICSI & $111(93.2 \%)$ & $114(91.2 \%)$ & \\
\hline Volume (mL) & $2.8(1.3)$ & $2.8(1.6)$ & 0.725 \\
\hline Concentration, $\mathrm{mil} / \mathrm{mL}$ & $66.1(43.8)$ & $79.3(64.4)$ & 0.064 \\
\hline Motility, \% & $44.3(22.5)$ & $45.3(22.6)$ & 0.726 \\
\hline Total motile count, mil & $99.6(107.4)$ & $108.3(109.9)$ & 0.535 \\
\hline Forward progression, $0-4$ score & $3.0(0.4)$ & $3.1(0.6)$ & 0.105 \\
\hline Fertilization rate, all (\%) & $77.6(21.1)$ & $77.9(21.0)$ & 0.939 \\
\hline Fertilization rate, IVF (\%) & $59.9(34.9)$ & $61.8(33.0)$ & 0.904 \\
\hline Fertilization rate, ICSI (\%) & $78.9(19.4)$ & $79.4(19.0)$ & 0.848 \\
\hline D5 usable quality blastocyst rate (\%) & $51.6(22.7)$ & $55.5(20.5)$ & 0.225 \\
\hline \multicolumn{3}{|l|}{ Day of transfer } & \multirow[t]{3}{*}{0.031} \\
\hline D3 transfer & $27(42.2 \%)$ & $19(24.7 \%)$ & \\
\hline D5 transfer & $37(57.8 \%)$ & $58(75.3 \%)$ & \\
\hline Number of embryos transferred & $1.7(0.8)$ & $1.7(0.7)$ & 0.760 \\
\hline Implantation rate $(\%)$ & $25.3(38.3)$ & $37.7(42.2)$ & 0.072 \\
\hline Pregnancy rate $(\%)$ & 48.4 & 56.7 & 0.343 \\
\hline
\end{tabular}

* Mean (SD) or $N(\%)$

\begin{tabular}{lll}
\hline & $\begin{array}{l}\text { Regression coefficient (95\% CI) } \\
\text { OR adjusted odds ratio (95\% CI) }\end{array}$ & $p$-value \\
\hline${\text { Motility, } \%^{\mathrm{a}}}^{\text {Total motile count, mil }}{ }^{\mathrm{a}}$ & $1.223(-4.552,6.998)$ & 0.677 \\
Forward progression, 0-4 score $^{\mathrm{a}}$ & $7.311(-20.505,35.127)$ & 0.605 \\
${\text { Fertilization rate }(\%)^{\mathrm{b}}}^{\text {D5 usable quality blastocyst rate }(\%)^{\mathrm{b}}}$ & $0.105(-0.025,0.235)$ & 0.112 \\
${\text { Pregnancy rate }(\%)^{\mathrm{c}}}$ & $0.001(-0.052,0.054)$ & 0.981 \\
& $0.038(-0.026,0.101)$ & 0.241 \\
\hline
\end{tabular}

${ }^{a}$ Linear regression model adjusted for paternal age and days of abstinence

${ }^{\mathrm{b}}$ Linear regression model adjusted for maternal age, paternal age, days of abstinence, and fertilization method

${ }^{\mathrm{c}}$ Logistic regression model adjusted for maternal age, paternal age, days of abstinence, fertilization method, number of embryos transferred, and day of transfer 


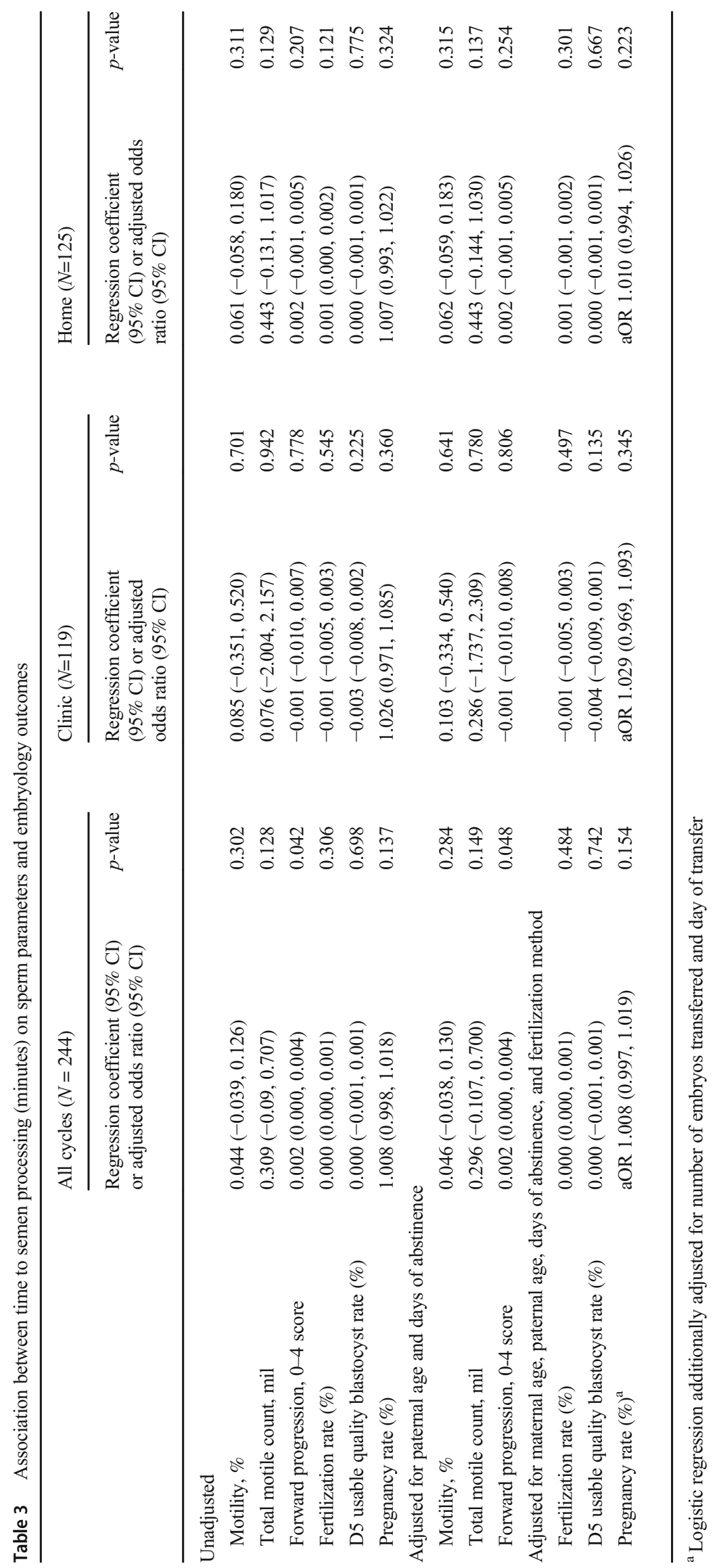


patients who underwent subsequent IVF cycles before and after COVID-19 (Table 4). The majority of patients underwent two cycles in less than a year. Mean male age was 38.1 years in the clinic group and 38.9 years in the home group $(p<0.001)$. On average, men were abstinent for 2.9 days (SD 1.3) in the clinic group and 3.3 days (SD 3.6) in the home group $(p=0.576)$. Mean time to semen processing was $34.0 \mathrm{~min}$ (SD 11.4) in the clinic group and $78.7 \mathrm{~min}$ (SD 28.5) in the home group $(p<0.001)$.

Sperm concentration, percent motility, total motile count, and forward progression score were similar between the clinic and home collections in this subgroup of patients (Table 4). While there was no change in sperm parameters by the amount of time to processing in clinic samples, those collected at home demonstrated an increase in motility of $0.36 \%(p=$ $0.002)$, an increase in total motile count by 1.6 million $(p=$ $0.007)$, and an increase in forward progression score of $0.01(p$ $=0.006)$ (Table 5 ). These findings persisted when controlling for paternal age and days of abstinence.

There were no differences in mean FRs (clinic 77.0\%, SD 22.5 vs. home $77.9 \%$, SD 18.2; $p=0.813$ ) or D5-UQBRs (clinic $47.2 \%$, SD 21.3 vs. home $54.6 \%$, SD 24.8; $p=$ 0.218 ). Longer time interval between semen production and processing had no effect on FRs or D5-UQBRs in the unadjusted model (Table 5). After adjusting for confounders, only an increase in fertilization rate of $0.006 \%$ ( $p=$ 0.038 ) in the clinic group with increased time to processing was observed (Table 5).

\section{Discussion}

The WHO laboratory manual states that semen specimens produced at home should be delivered to the andrology laboratory for processing within $1 \mathrm{~h}$ and be maintained between 20 and $40^{\circ} \mathrm{C}$ during that time [1]. Reduced sperm motility and viability have been observed over time and at temperature extremes, perhaps due to the accumulation of non-sperm components, such as bacteria or reactive oxygen species $[2-4,16$, 17]. Importantly, clinic collection, the recommended standard of care for diagnostic samples, maximally ensures the safety and identity of the semen sample. However, the exceptional needs for social distancing during the COVID-19 pandemic have prompted many changes in routine fertility care. Home semen collection is a valuable tool that can enable increased social distancing and patient convenience. Thus, it is crucial to understand whether home semen collection and time over $1 \mathrm{~h}$ for travel between collection and processing negatively affects couples' chances of achieving a usable blastocyst and positive pregnancy test.

Prior studies have evaluated how location of semen collection and length of time from semen collection to processing, with the assumed greater duration and fluctuations in temperature during transport, impact semen analysis parameters and IUI outcomes, with mixed findings [5-10, 18]. In the randomized trial conducted by Gao et al., semen analysis results were similar between men who collected at home and at clinic, and patient satisfaction was higher after home collection [6]. Reassuringly, sperm morphology and levels of DNA fragmentation were also similar between the two groups. However, time to processing was not assessed, and these men were not undergoing fertility treatment. In the sole study to our knowledge evaluating semen parameters and embryo outcomes in a large cohort of couples undergoing IVF/ICSI who collected at home versus clinic, the patients chose their collection site and confounders such as maternal age and fertilization method were not controlled [8]. Given the limitations in method and scope of the literature, we aimed to evaluate how both collection location and time between collection and processing affect sperm parameters and embryology outcomes for couples undergoing IVF/ICSI in the exceptional context of the COVID-19 pandemic. Our study suggests that
Table 4 Baseline characteristics and semen analysis parameters of couples with cycles both prior to and after COVID-19*

\begin{tabular}{llll}
\hline & Clinic $(N=42)$ & Home $(N=42)$ & $p$-value \\
\hline Age female, yrs & $37.4(3.8)$ & $38.2(3.7)$ & $<0.001$ \\
Age male, yrs & $38.1(5.3)$ & $38.9(5.1)$ & $<0.001$ \\
Days of abstinence & $2.9(1.3)$ & $3.3(3.6)$ & 0.546 \\
Time to processing, min & $34.0(11.4)$ & $78.7(28.5)$ & $<0.001$ \\
Volume (mL) & $3.0(1.5)$ & $2.9(1.5)$ & 0.801 \\
Concentration, mil/mL & $78.6(40.9)$ & $81.0(51.5)$ & 0.730 \\
Motility, \% & $52.3(22.8)$ & $49.7(21.8)$ & 0.152 \\
Total motile count, mil & $127.8(101.6)$ & $123.1(111.3)$ & 0.730 \\
Forward progression, 0-4 score & $3.0(0.6)$ & $3.1(0.7)$ & 0.200 \\
Fertilization rate $(\%)$ & $77.0(22.5)$ & $77.9(18.2)$ & 0.813 \\
D5 usable quality blastocyst rate $(\%)$ & $47.2(21.3)$ & $54.6(24.8)$ & 0.218 \\
\hline
\end{tabular}

*Mean (SD) 


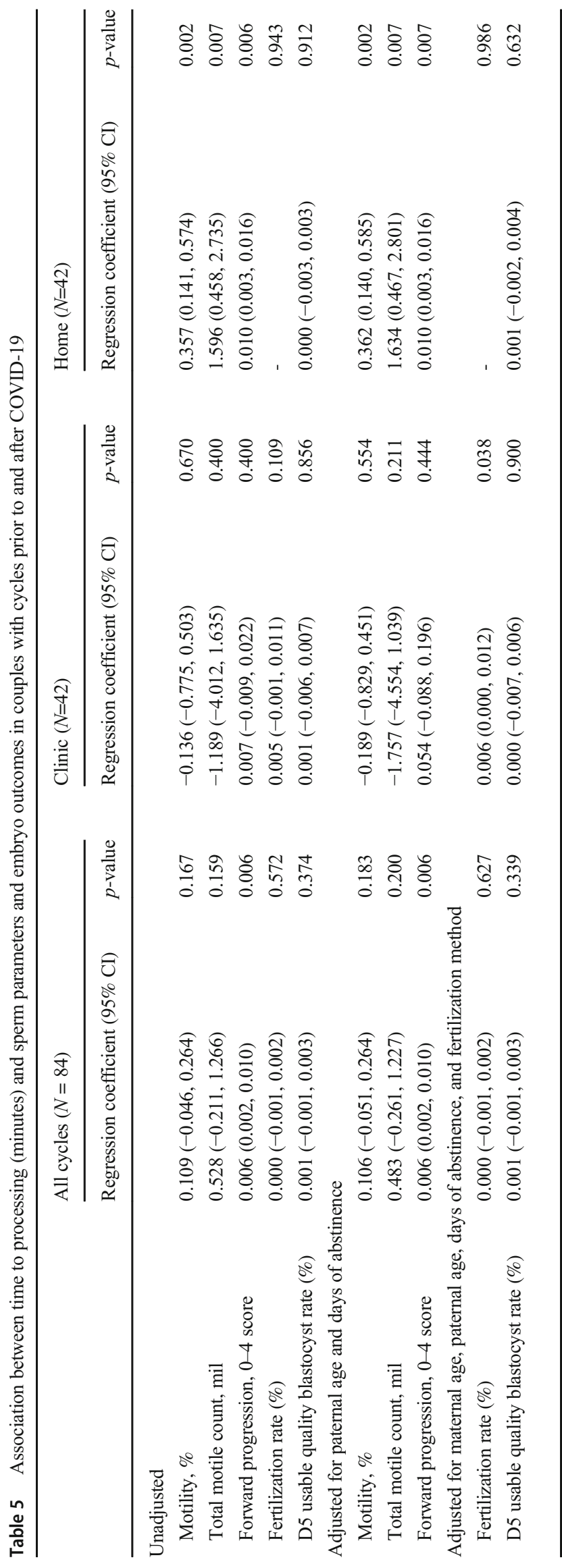


neither home collection nor processing within $2 \mathrm{~h}$ of collection is negatively associated with sperm parameters, FR, D5UQBR, or PR.

Consistent with several other studies, we observed no detrimental effects of home collection, in our total cohort or within the same patient. Amongst conventional IVF cycles, for which changes in motility might have a greater detrimental effect on fertilization, we observed no differences in fertilization rate between the home and clinic groups. While the WHO manual recommends processing within $1 \mathrm{~h}$ for home collection of semen analyses, Appell et al. found that the most significant declines in sperm motility and viability at 20 and $37^{\circ} \mathrm{C}$ occurred beginning 3-6 $\mathrm{h}$ after collection $[1,3]$. Our findings reinforce these early observations and suggest that motility remains relatively constant at or near body temperature for at least up to $2 \mathrm{~h}$. Any slight changes in motility or sperm quality do not appear to affect early IVF outcomes. On the other hand, reduced stress at home, a proposed reason for superior quality of home collections [7, 19], or positive lifestyle changes during the COVID-19 quarantine, did not improve sperm parameters in our cohort of patients. Of note, we observed a significant increase in forward progression score with longer time to processing in all cycles in both unadjusted and adjusted models. Although statistically significant, an increase in 0.002 progression score is likely not clinically meaningful. This finding may be attributed to the decrease in viscosity of seminal fluid over time [20]. Additionally, in our subgroup of patients with pre- and post-COVID-19 cycles, we observed a marginal increase in fertilization rate with increasing time in the clinic group, as well as a slight but statistically significant improvement in motility, total motile count, and forward progression score with increasing time after home collection, even after controlling for confounding variables. A positive association with time, due to a factor such as reduced semen viscosity, should also be present in both groups, suggesting these observations are likely an artifact of low power. Ultimately, there was no difference in the D5-UQBR. Therefore, more studies are needed to update the literature on how sperm parameters vary over time under specific conditions.

This study has several key strengths. Our IVF laboratory implemented a single protocol change due to the COVID-19 pandemic that allowed us to evaluate the effect of semen collection location and time to processing on sperm parameters and embryology outcomes while ensuring that all other conditions remained consistent between the clinic and home groups over a short period of time. We believe this design reduced the risk of selection bias, though most couples who underwent cycles immediately after the COVID-19 shutdown were potentially lower prognosis individuals intent on restarting fertility treatment as soon as possible. Home collections were all performed with masturbation using the standard collection cup, which avoided the additional confounder of interrupted coitus, which was allowed in the study by Shetty Licht et al. [5]. Our analyses took other key potential confounders into account, including days of abstinence, as this duration has been shown to alter sperm parameters and was not consistently controlled in prior studies [5, 7, 21-23]. Another strength of this study is the subgroup analysis of patients with a cycle using clinic collection and a cycle using home collection, illustrating that within the same patient, there was no detrimental effect of home collection. Because these patients served as their own control, this reduced the likelihood of uncontrollable patient-specific variables affecting our results.

The cycles included in our analysis were collected during 2020, and while we included PR data, complete clinical pregnancy and live birth rate data were not available. Our cohort included a small number of conventional IVF cycles, and as fertilization in these cycles may be more susceptible to deterioration in sperm motility or quality, further investigation with a larger number of conventional IVF cycles is warranted. Future studies should also explore whether semen collection and processing conditions have any long-term impact on the offspring in IVF/ICSI cycles. For our subgroup of patients who underwent a cycle prior to and after COVID-19, we did not include a PR comparison, as the majority of these patients underwent a short interval repeat IVF cycle after an unsuccessful prior attempt. The times to processing for home collections varied significantly, which may have reduced our ability to detect certain differences between the home and clinic groups. It is also possible that our home collection findings are not generalizable to other fertility clinic populations or to other seasons (i.e., winter travel conditions). However, given the range in commuting distances across a large metropolitan city that include walking, driving, and train travel, we believe that our population captures a variety of remote collection settings. Furthermore, Song and colleagues found no impact of season on home collection IUI outcomes in their study [10]. Finally, further studies could assess how longer time periods up to $6 \mathrm{~h}$ between collection and processing affect sperm parameters and early IVF outcomes.

\section{Conclusion}

In conclusion, semen collection location and increased time to sperm processing up to $2 \mathrm{~h}$ have had no negative impact on sperm parameters, FR, D5-UQBR, or PR in our clinic population. If a male patient can deliver the sample himself to ensure proper identification, our findings suggest that couples undergoing IVF/ICSI can be given the option routinely to collect their semen sample at home as long as they live within $2 \mathrm{~h}$ of their fertility clinic. This work contributes practical and reassuring data on home semen collection for IVF/ICSI cycles that can increase patient safety and flexibility during the ongoing COVID-19 crisis. 
Acknowledgements The authors acknowledge the MGH embryologists for their extraordinary efforts to ensure patient safety.

Availability of data and material Not applicable.

Code availability Not applicable.

Author contribution CRS conceived of the project, interpreted the data, and drafted the manuscript. SV performed the data analysis and interpreted the data. CLB collected the data and assisted in its interpretation. All authors reviewed and edited the final manuscript.

\section{Declarations}

Ethics approval The study was approved by Partners Healthcare Institutional Review Board at Massachusetts General Hospital.

Consent to participate This was an IRB-approved retrospective cohort study

Consent for publication Not applicable. Study data was de-identified.

Conflict of interest The authors declare no competing interests.

\section{References}

1. WHO laboratory manual for the examination and processing of human semen. Fifth ed. Geneva, Switzerland: WHO Press; 2010.

2. Aitken RJ. Oxidative stress in the male germ line and its role in the aetiology of male infertility and genetic disease. Reprod BioMed Online. 2003;7(1):65-70.

3. Appell RA, Evans PR. The effect of temperature on sperm motility and viability. Fertil Steril. 1977;28(12):1329-32.

4. Griveau JF, Le Lannou D. Reactive oxygen species and human spermatozoa: physiology and pathology. Int J Androl. 1997;20: 61-9.

5. Shetty Licht R, Handel L, Sigman M. Site of semen collection and its effect on semen analysis parameters. Fertil Steril. 2008;89(2): 395-7. https://doi.org/10.1016/j.fertnstert.2007.02.033.

6. Gao J, Duan YG, Yi X, Yeung WSB, Ng EHY. A randomised trial comparing conventional semen parameters, sperm DNA fragmentation levels and satisfaction levels between semen collection at home and at the clinic. Andrologia. 2020;52(7). https://doi.org/10. 1111/and.13628.

7. Elzanaty S, Malm J. Comparison of semen parameters in samples collected by masturbation at a clinic and at home. Fertil Steril. 2008;89(6):1718-22. https://doi.org/10.1016/j.fertnstert.2007.05. 044.

8. Stimpfel M, Jancar N, Vrtacnik-Bokal E. Collecting a semen sample at home for an ART procedure positively affects the blastocyst and embryo utilization rate. Reprod BioMed Online. 2020;42:3919. https://doi.org/10.1016/j.rbmo.2020.09.021.

9. Yavas Y, Selub MR. Intrauterine insemination (IUI) pregnancy outcome is enhanced by shorter intervals from semen collection to sperm wash, from sperm wash to IUI time, and from semen collection to IUI time. Fertil Steril. 2004;82(6):1638-47. https:// doi.org/10.1016/j.fertnstert.2004.04.061.

10. Song GJ, Herko R, Lewis V. Location of semen collection and time interval from collection to use for intrauterine insemination. Fertil
Steril. 2007;88(6):1689-91. https://doi.org/10.1016/j.fertnstert. 2007.01.051.

11. Sacha CR, Chavarro JE, Williams PL, Ford J, Zhang L, Donahoe $\mathrm{PK}$, et al. Follicular fluid anti-Müllerian hormone (AMH) concentrations and outcomes of in vitro fertilization cycles with fresh embryo transfer among women at a fertility center. J Assist Reprod Genet. 2020;37(11):2757-66. https://doi.org/10.1007/s10815-02001956-7.

12. Sacha CR, Harris AL, James K, Basnet K, Freret TS, Yeh J et al. Placental pathology in live births conceived with in vitro fertilization after fresh and frozen embryo transfer. American journal of obstetrics and gynecology. 2019.

13. Souter I, Smith KW, Dimitriadis I, Ehrlich S, Williams PL, Calafat $\mathrm{AM}$, et al. The association of bisphenol-A urinary concentrations with antral follicle counts and other measures of ovarian reserve in women undergoing infertility treatments. Reprod Toxicol. 2013;42: 224-31. https://doi.org/10.1016/j.reprotox.2013.09.008.

14. Chavarro JE, Ehrlich S, Colaci DS, Wright DL, Toth TL, Petrozza $\mathrm{JC}$, et al. Body mass index and short-term weight change in relation to treatment outcomes in women undergoing assisted reproduction. Fertil Steril. 2012;98(1):109-16. https://doi.org/10.1016/j. fertnstert.2012.04.012.

15. Chavarro JE, Ehrlich S, Colaci DS, Wright DL, Toth TL, Petrozza $\mathrm{JC}$, et al. Body mass index and short-term weight change in relation to treatment outcomes in women undergoing assisted reproduction. Fertil Steril. 2012;98(1):109-16.

16. Aitken RJ, Krausz C. Oxidative stress, DNA damage and the $\mathrm{Y}$ chromosome. Reproduction. 2001;122:497-506.

17. Agarwal A. Role of antioxidants in treatment of male infertility: an overview of the literature. Reprod BioMed Online. 2004;8(6):61627.

18. Lemmens L, Kos S, Beijer C, Braat DDM, Nelen WLDM, Wetzels AMM. Techniques used for IUI: is it time for a change? Hum Reprod. 2017;32(9):1835-45. https://doi.org/10.1093/humrep/ $\operatorname{dex} 223$.

19. Clarke RN, Kolck SC, Geoghegan A, Travassos DE. Relationship between psychological stress and semen quality among in-vitro fertilization patients. Hum Reprod. 1999;14(3):753-8.

20. Ooi EH, Smith DJ, Gadêlha H, Gaffney EA, Kirkman-Brown J. The mechanics of hyperactivation in adhered human sperm. Royal Soc Open Sci. 2014;1(2):140230. https://doi.org/10.1098/ rsos. 140230.

21. Levitas E, Lunenfeld E, Weiss N, Friger M, Har-Vardi I, Koifman $\mathrm{A}$, et al. Relationship between the duration of sexual abstinence and semen quality: anaysis of 9489 semen samples. Fertil Steril. 2005;83(6):1680-6. https://doi.org/10.1016/j.fertnstert.2004.12. 045 .

22. Marshburn PB, Giddings A, Causby S, Matthews ML, Usadi RS, Steuerwald N, et al. Influence of ejaculatory abstinence on seminal total antioxidant capacity and sperm membrane lipid peroxidation. Fertil Steril. 2014;102(3):705-10. https://doi.org/10.1016/j. fertnstert.2014.05.039.

23. Alipour H, Van Der Horst G, Christiansen OB, Dardmeh F, Jørgensen N, Nielsen HI, et al. Improved sperm kinetics in semen samples collected after $2 \mathrm{~h}$ versus 4-7 days of ejaculation abstinence. Hum Reprod. 2017;32(7):1364-72. https://doi.org/10. 1093/humrep/dex101.

Publisher's note Springer Nature remains neutral with regard to jurisdictional claims in published maps and institutional affiliations. 